\title{
Voting behavior in one-shot and iterative multiple referenda
}

\author{
Umberto Grandi* Jérôme Lang ${ }^{\dagger} \quad$ Ali Ozkes ${ }^{\ddagger}$ \\ Stéphane Airiau ${ }^{\S}$
}

December 12, 2020

\begin{abstract}
We consider a set of voters making a collective decision via simultaneous vote on two binary issues. Voters' preferences are captured by payoffs assigned to combinations of outcomes for each issue and they can be nonseparable: a voter's preference over an issue might be dependent on the other issue. When the collective decision in this context is reached by voting on both issues at the same time, multiple election paradoxes may arise, as studied extensively in the theoretical literature. In this paper we pursue an experimental approach and investigate the impact of iterative voting, in which groups deliberate by repeating the voting process until a final outcome is reached. Our results from experiments run in the lab show that voters tend to have an optimistic rather than a pessimistic behaviour when casting a vote on a non-separable issue and that iterated voting may in fact improve the social outcome. We provide the first comprehensive empirical analysis of individual and collective behavior in the multiple referendum setting.
\end{abstract}

Keywords: Behavioural social choice · Deliberation · Iterative voting - Multiple referenda · Voting on combinatorial domains

JEL Codes: C91 · C92 - D71 - D72 - D92

\footnotetext{
*IRIT, Université Toulouse 1 Capitole, France, E-mail: umberto.grandi@irit.fr

${ }^{\dagger}$ CNRS, LAMSADE, Université Paris-Dauphine, PSL, France, E-mail: lang@lamsade.dauphine.fr

${ }^{\ddagger}$ Wirtschaftsuniversität Wien, Institute for Markets and Strategy, Vienna, Austria. E-mail: ali.ozkes@wu.ac.at

${ }^{\S}$ LAMSADE, Université Paris-Dauphine, PSL, France, E-mail: airiau@lamsade.dauphine.fr
} 


\section{Introduction}

The deliberative view of collective choice aims at designing protocols for decision makers to reach a consensus by informing each others of their preferences. While deliberation in collective decision-making mostly focuses on contexts with few agents, recent works advocate deliberative approaches for reaching collective decisions among large group of agents.

In a first series of approaches, group deliberation is performed via repeated deliberation among small groups of agents. In (Goel and Lee, 2016), each stage consists of three people deliberating and designating a fourth one to whom they grant their votes; the process goes on until all votes are accumulated on a single remaining individual. Fain et al. (2017) define a sequential deliberation protocol where at each stage, a randomly selected pair of agents bargain over the decision space using as disagreement point the alternative that was obtained in the previous stage. In (Garg et al., 2019) a voter is randomly selected at each stage and asked to modify a candidate solution within some local neighborhood of its current value. Elkind et al. (2020) describe and study a deliberation process in which agents dynamically form coalitions supporting proposals that they prefer over the status quo.

A second another important line of work pertains to iterative voting. While voting is traditionally seen as a centralized process (voters report their votes to a central authority, which then determines the outcome), a very active, recent research line, starting with the seminal work of Meir et al. (2010), argues that better outcomes can be reached if voters are allowed to change their vote a number of times, after seeing the distribution of the others' votes. ${ }^{1}$ Voting is thus seen as a sequential deliberation protocol, where each deliberation stage consists of a single voter or all voters (depending on the framework) recasting their votes in view of the current distribution. Such protocols, unlike classical deliberation protocols in social choice, make sense also for large groups of voters.

Most work on iterative voting consists of theoretical analyses of singlewinner iterative voting, such as convergence and efficiency guarantees. A noticeable exception is the analysis of behavioural online experiments (still for single-winner voting) by Meir et al. (2020), who investigate the distribution of types among voters based on a number of myopic heuristics, and compare one-shot voting with access to an information poll to iterative voting where voters can also see each others' votes.

Beyond single-winner voting, a context where iterative voting can be (even more) helpful for the voters to coordinate and reach better outcomes (and thus, in a sense, vote strategically in the positive sense of the term), is multiple referenda: voters have to come up with a collective yes/no decision

\footnotetext{
${ }^{1}$ For a survey, see Meir (2017); note that a number of papers have appeared since then.
} 
on a number of binary issues, upon which they may have nonseparable preferences. Such situations give rise to a number of problems such as those presented in the following example:

Example 1. Three voters need to decide on two binary issues (which can represent, for instance, the construction of two public goods, such as a swimming pool and a tennis court). We denote by $Y$ and $N$ the two possible values for each of the issues. Each issue is decided by a majority vote, with voters expressing their vote simultaneously on both issues. Voters have the following preferences on the four possible outcomes:

\begin{tabular}{l|ccccccc} 
Voter 1 & $Y Y$ & $\succ$ & $Y N$ & $\succ$ & $\succ$ & $N N$ \\
Voter 2 & $N Y$ & $\succ$ & $N N$ & $\succ$ & $\succ$ & $\succ Y$ \\
Voter 3 & $Y N$ & $\succ$ & $N Y$ & $\succ$ & $N N$ & $\succ$ & $Y Y$
\end{tabular}

Note that voter 1's preference on the first issue does not depend on the outcome of the second issue: she prefers a $\mathrm{Y}$ outcome to an $\mathrm{N}$ outcome, irrespective of the result of the second issue. Symmetrically, her preference on the second issue does not depend on the first: she has separable preferences. This is however not the case for the other two voters: voter 2's preference on the second issue depends on the value of the first one (she prefers outcome $\mathrm{Y}$ on the second issue if the first issue takes value $\mathrm{N}$, and $\mathrm{N}$ if it takes value $\mathrm{Y}$ ) and voter 3's preferences on each of the issues depend on the value of the other one.

If we assume that the three voters vote for their favourite combination, the result of majority vote will be YY, which is the worse combination for two out of three voters. Such problems are known in the literature as multiple election paradoxes (Brams et al., 1998). Designing ways to avoid multiple election paradoxes is known as a very difficult problem (see Section 2 for details). Perhaps the most convincing method we know of (at least in some contexts) is the one suggested by Bowman et al. (2014): allowing for iterative voting. They run computer simulations on a number of voting profiles with two and three binary issues giving rise to multiple election paradoxes, giving to the voters the possibility of changing their votes in an iterated process. Their results show that iteration leads to a statistically significant improvement (see Section 2 for details).

However, the extent to which the results based on simulations would carry to the real world is not clear. This is the aim of our paper: we propose lab experiments to investigate the behavior of human subjects in the context of multiple referenda and the impact of iterative voting on both individual and collective behavior. For the sake of simplicity, we focus on two binary issues. Our conclusions are twofold. First, we find out that iterative voting does improve the quality of the outcome, confirming the conclusions of Bowman et al. (2014); second, we analyse the way voters vote in presence of nonseparability, both in one-shot and iterative voting scenarios: they 
tend to vote rationally (in a sense to be defined in the sequel), and to vote optimistically more frequently than pessimistically.

The paper is organised as follows. In Section 2 we discuss in detail the related literature on multiple referenda and iterative voting. Section 3 presents the formal setting and Section 4 the details of our experiments. We then present results on the optimistic and pessimistic behaviour of voters (Section 5), on the individual voting dynamics in iteration (Section 6), and on the evolution of global parameters in iteration (Section 7). Section 8 concludes the paper. The two appendices are devoted to some further experimental findings (A) and experimental instructions (B).

\section{Related work}

In this section we survey related work on which our experimental setting is grounded.

\subsection{Preferences on combinatorial domains, and multiple ref- erenda}

A combinatorial domain is a Cartesian product of finite value domains: $A=D_{1} \times \ldots \times D_{p}$, where for each $i, D_{i}$ is the value domain for a variable $X_{i}$. The paradigmatic example is a set of binary propositions (or issues) upon which voters have to make a yes/no decision. A strict preference relation $\succ$ over $A$ is an asymmetric and transitive relation. Variable $X_{i}$ is said to be separable for $\succ$ if preferences over values in $D_{i}$ are the same whatever the values of other variables. The preference relation $\succ$ is separable if all variables are separable for it. The structural properties and the combinatorics of such relations has been investigated in several papers, such as Bradley et al. (2005) and Hodge et al. (2009). The prohibitive size of combinatorial domains often prevents voters from expressing a preference relation explicitly (by listing all alternatives in the preference order). This has lead a number of researchers to develop tools for representing such preferences succinctly.

A multiple referendum consists of taking a collective decision over a combinatorial domain with binary variables, called issues. ${ }^{2}$ Nonseparability is a huge problem in multiple referenda: on the one hand, letting voters vote individually on each variable ignores their preferential dependencies between issues and may lead to bad collective decisions; on the other hand, letting agents express their preferences over all combinations of values is not feasible due to the size of the domain, as soon as the number of issues exceeds a few units. This dilemma has been identified for a long time (Brams et al., 1997,

\footnotetext{
${ }^{2}$ Examples from the real world are ubiquitous, including the commonly cited decisionmaking processes in Switzerland and California. For the latter, for instance, see: https: //ballotpedia.org/California_2020_ballot_propositions.
} 
1998; Lacy and Niou, 2000). Since then, a number of solutions has been suggested and studied. They are reviewed by Lang and Xia (2016), who conclude that "After reviewing several classes of methods (...), we are left with the (expected) conclusion that none of them is perfect." We give here a brief summary of these classes of methods, together with their pros and cons:

- simultaneous voting: all voters vote for each issue separately, and majority rule is used to decide on each issue independently. This is the simplest solution, as it is very cheap in communication and (to a lesser extent) cognitive effort; it is used in most occurrences of multiple referenda, such as in Switzerland or California. However, this simple setting is very much prone to severe paradoxes (Brams et al., 1998; Lacy and Niou, 2000), and the outcome may be far from the optimum. ${ }^{3}$ The existence and characterization of equilibria for simultaneous voting, formulated as a Bayesian game, is studied by Ahn and Oliveros (2012).

- sequential voting: voters vote on individual issues in sequence; they can see the outcome on earlier issues when they vote on a later issue. Sequential voting partly resolves some of the problems of simultaneous voting, and remains relatively cheap in communication, but it is still prone to paradoxes (Lang and Xia, 2009; Cornelio et al., 2019).

- expressing preferences in a compact language: voters express their preferences in a sophisticated language whose aim is to offer a trade-off between succinctness and expressiveness, such as CP-nets (Conitzer et al., 2011), conditionally lexicographic preference trees (Lang et al., 2018), or graphical languages for cardinal preferences (Gonzales et al., 2008). This allows a very expressive expression of preferences, henceforth to an outcome of reasonably good quality, but unfortunately it comes with an important burden on the voters in terms of communication and cognitive effort.

- expressing preference as distance to the top alternative: voters express only their most preferred alternative, and their whole preference relation is then induced from a predefined distance between alternatives, such as the Hamming distance (Brams et al., 2007; Çuhadaroğlu and Lainé, 2012). While this is reasonable in some contexts, it fails to express nonseparability and hence will perform badly in contexts where voters have nonseparable preferences.

Another solution, which is suggested by Bowman et al. (2014), stands out as structurally different than the ones cited above. It consists of an

\footnotetext{
${ }^{3}$ Paradoxes arise even if voters have separable preferences, as pointed out by Benoît and Kornhauser (2010) as well as by Özkal-Sanver and Sanver (2006).
} 
iterative voting protocol that allows voters to revise their votes based on the outcome of previous iterations. It allows to reach a solution of reasonably good quality, while keeping the communication and cognitive burden at a reasonable level. We will describe this solution in detail after our discussion of iterative voting in Section 2.2.

\section{$2.2 \quad$ Iterative voting}

The idea of reaching equilibria by means of iterated processes has a long history in economics (for a survey see, e.g., Laffont, 1987), but it is only in recent years that iterative voting became an established setting on the research agenda in computational social choice.

Three research lines arose in this setting. First, iterative voting can be used for modelling voter response to polls preceding an election. Under this interpretation, convergence issues have been investigated (see, e.g., Meir et al., 2010, 2017; Lev and Rosenschein, 2016), as well as the possibility of manipulation by the central authority (Wilczynski, 2019; Baumeister et al., 2020). Second, iterative voting methods can be designed as voting rules per se (Grandi et al., 2013; Obraztsova et al., 2015; Airiau et al., 2017). Third, iterative voting has been used as a tool to investigate strategic voting under uncertainty, with partial information coming either from an exogenous poll or by making the votes of the other voters visible in iterated elections (Meir et al., 2020). With very few exceptions, most results in iterative voting assume that only one individual at a time is allowed to change their vote.

We envisage iterative voting as a deliberative method to allow for informed votes. In our setting, all voters are allowed to change their vote at the same time (which is an important contrast with most of the literature in iterative voting) and can observe the current outcome; such observations constitute a reliable source of information for the voters who are then able to learn (if partially) each others' preferences and behaviour and adjust their votes accordingly. At the same time, investigating the behaviour of voters in the initial and subsequent stages of the iterative voting process allows to draw conclusions on individual voting behaviour under various levels of uncertainty.

Our model is based on the work of Bowman et al. (2014), who devised comprehensive computer simulations to show that a simple iterative voting protocol can be used to help voters get out of multiple election paradoxes. The authors focus on three-issue multiple referenda and select a set of preference profiles in which sincere simultaneous majority voting leads to particularly unsatisfactory results. They run extensive computer simulations on combinations of such profiles, with voters participating in an iterated election with the possibility of changing their vote at each iteration step. Each voter is programmed so as to choose, at each iteration step, the vote that maximises a given score, determined from the sincere preferences 
of the voter, an attainability factor representing the likelihood of a vote outcome based on past iteration results, and a learning factor indicating actions that previously yielded desirable results. Their results show an improvement of the average score index (ASI), i.e., the average Borda score of the winning combination, in almost all considered cases. Similar albeit preliminary results have also been obtained by Liekah (2019) using learning agents programmed with reinforcement learning in a similar setting.

\section{Context and setting}

\subsection{Classes of preferences and associated behaviours}

Consider a combinatorial domain made of two binary variables, called Row and Col, with $D_{\text {Row }}=\{$ Top, Bottom $\}$ and $D_{\text {Col }}=\{$ Left, Right $\}$. Choosing to restrict the study to two variables is, admittedly, a loss of generality. We made this choice so as to limit as much as possible the burden of cognitive effort: three binary variables involve reasoning with eight values, which could be difficult for many subjects. The extent to which this is a loss of generality will be discussed in Section 8 .

The set of alternatives, called cells, is thus

$$
A=\{(\text { Top, Left), (Top, Right), (Bottom, Left), (Bottom, Right) }\}
$$

which we denote more simply by $A=\{T L, R T, B L, B R\}$.

A (strict) preference relation $\succ$ over $A$ is a transitive and asymmetric relation. If it is moreover complete, it is said to be linear. We say that Col is separable for $\succ$ if (TR $\succ T L$ if and only if $B R \succ B L)$ and $(T L \succ T R$ if and only if $B L \succ B R) .{ }^{4}$ Likewise, Col is separable for $\succ$ if $T R \succ B R$ holds if and only if $T L \succ B L$ and $B R \succ T R$ holds if and only if $B L \succ T L$. If both Col and Row are separable for $\succ$ then $\succ$ is said to be separable; if only one of $\mathrm{Col}$ and Row is separable for $\succ$ then $\succ$ is said to be semi-separable; if none of Col and Row is separable for $\succ$ then $\succ$ is said to be fully nonseparable. When we say that a preference is non-separable we mean that it is either semi-separable or fully non-separable.

The restriction to two binary variables makes it very easy to count the number of linear preference relations in each class: ${ }^{5}$

- separable: $T R \succ T L \succ B R \succ B L$, and the seven other linear preference relations obtained by exchanging the role of Col and Row and/or permuting one of the values, or both, of Col and Row.

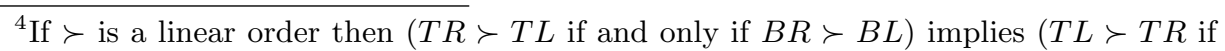
and only if $B L \succ B R$ ).

${ }^{5}$ The restriction to two variables is crucial: counting the number of separable preferences is an open problem.
} 
- semi-separable: $T R \succ T L \succ B L \succ B R$, and the seven other linear preference relations obtained by applying a permutation as above.

- fully non-separable: $T R \succ B L \succ B R \succ T L$, and the seven other linear preference relations obtained by applying a permutation as above.

\subsection{Individual preferences as utility matrices}

We provide voters with utility matrices, with the two rows representing the two possible outcomes of the vote on the first issue (Top or Bottom) and the column the second issue (Left or Right). Below we show three utility matrices, corresponding to preference relations that are respectively separable, semi-separable, and fully non-separable.

\begin{tabular}{r|c|c|}
\multicolumn{1}{c}{} & \multicolumn{1}{c}{ Left } & Right \\
\cline { 2 - 3 } Top & 3 & 2 \\
\cline { 2 - 3 } Bottom & 1 & 0 \\
\cline { 2 - 3 } & &
\end{tabular}

\begin{tabular}{l|l|l|}
\multicolumn{1}{c}{} & \multicolumn{1}{l}{$\mathrm{L}$} & \multicolumn{1}{l}{$\mathrm{R}$} \\
\cline { 2 - 3 } $\mathrm{T}$ & 3 & 0 \\
\cline { 2 - 3 } $\mathrm{B}$ & 2 & 1 \\
\cline { 2 - 3 } & &
\end{tabular}

\begin{tabular}{|c|c|c|}
\hline & $\mathrm{L}$ & $\mathrm{R}$ \\
\hline $\mathrm{T}$ & 0 & 3 \\
\hline $\mathrm{B}$ & 2 & 1 \\
\hline
\end{tabular}

\subsection{Voter behaviour}

With each of the three types of linear preference relations, there are a number of possible associated behaviours:

1. Assume variable $V$ is separable for $\succ$, with $x_{V}$ as preferred value. Then $x_{V}$ is a dominating action in the sense that the agent can never be worse off by changing $\bar{x}_{V}$ into $x_{V}$ in her vote. We say that such a voter, in a given situation, is $V$-rational if she votes for $x_{V}$. For instance, the agent with the separable preference $T R \succ T L \succ B R \succ B L$ who votes for $(T, L)$ is Row-rational but Col-irrational.

2. An agent with a separable preference is said to be rational if she is both Col-rational and Row-rational, half-rational if she is rational for exactly one variable, and fully irrational if she is irrational for both. (The voter of the previous item is half-rational.) ${ }^{6}$

3. Assume an agent has the semi-separable preference (without loss of generality) $T R \succ T L \succ B L \succ B R$. The value $R$ of the non-separable variable $\mathrm{Col}$ is said to have a large span: if the voter is pivotal for this variable then it will lead to change the outcome either in favour of her best or of her worst alternative. Similarly, $L$ is said to have a small

\footnotetext{
${ }^{6}$ If two half rational voters have the preference relation $T R \succ T L \succ B R \succ B L$, it makes sense to distinguish further between one who votes for $L$ and one who votes for $B$ - the latter being perhaps more irrational than the former - but we will neglect this distinction.
} 
span. The voter is said to be optimistic if she votes for the large-span value and pessimistic otherwise. ${ }^{7}$

4. Assume an agent has the fully non-separable preference (without loss of generality) $T R \succ B L \succ B R \succ T L$. Variable $T$ has large span and $B$ has small span, therefore we also say that such a voter is optimistic (resp. pessimistic) if she votes for $T$ (resp. B). For Col, this is different: the value $R$ leads to either the best or the second worst alternative in case the voter is pivotal for Col, while $L$ leads to either the second best or the second worst alternative. We say that $R$ is stochastically dominant. The voter is said to be $S D$-rational if she votes for the stochastically dominant variable, and SD-irrational otherwise.

An example of each voter behaviour described above can be found in Figure 1 using the representation with utility matrices.

\begin{tabular}{|c|c|c|c|c|c|}
\hline & L (rat.) & $\mathrm{R}$ (irrat.) & & L (SD-rat.) & R (SD-irrat.) \\
\hline \multirow{2}{*}{$\begin{array}{r}\mathrm{T} \text { (rat.) } \\
\mathrm{B} \text { (irrat.) }\end{array}$} & 3 & 1 & \multirow{2}{*}{$\begin{array}{r}\mathrm{T} \text { (opt.) } \\
\mathrm{B} \text { (pess.) }\end{array}$} & 3 & 0 \\
\hline & 2 & 0 & & 1 & 2 \\
\hline
\end{tabular}

(a) Separable preference

(b) Semi-separable preference

\begin{tabular}{c|c|c|}
\multicolumn{1}{c}{} & \multicolumn{1}{c}{$\mathrm{L}$ (SD-rat.) } & $\mathrm{R}$ (SD-irrat.) \\
\cline { 2 - 3 } $\mathrm{T}$ (optimistic) & 3 & 0 \\
\cline { 2 - 3 } $\mathrm{B}$ (pessimistic) & 1 & 2 \\
\cline { 2 - 3 } & &
\end{tabular}

(c) Fully non-separable preference

Figure 1: Visual explanation of voter behaviour with three types of preferences over two issues.

\subsection{Simultaneous and iterative voting for multiple referenda}

A multiple referendum consists of a set of $p$ binary issues, and a set $N$ of $n$ voters, each of whom has private preferences over all combinations of issues. Simultaneous voting consists of asking each voter to cast a vote regarding each issue. Thus, for the two-issue case $(p=2)$, each of the $n$ voters has to vote twice: one for the column issue (that is, she has to vote for $L$ or for $R$ ) and one for the row issue (that is, she has to vote for $T$ or for $B$ ). When a voter casts a vote regarding an issue, she does not know the other votes regarding this issue, nor the votes regarding the other issue. For each issue, the collective decision is made via majority rule; from now on we assume $n$ to be odd so that ties cannot occur.

\footnotetext{
${ }^{7}$ Note that it is not necessary to write Col-optimistic, Row-optimistic, and so on, because there is only one variable for which this distinction applied; the other variable is separable.
} 
Iterative voting for multiple referenda is defined as a series of simultaneous voting stages; at each stage, voters observe the distribution of votes regarding each issue at the previous stage. Thus, at stage 1 , voters do not observe anything; at stage 2, they know the number of votes for $L, R, T$ and $B$ for stage 1 ; at stage 3 , they know the number of votes for $L, R, T$ and $B$ for stages 1 and 2 , and so on. The stopping criterion is defined exogeneously.

In order to evaluate the quality of the outcome, we focus on the average social welfare of the social outcome (the average payoff voters get) and the frequency of Condorcet winners being elected (an outcome $x$ is a Condorcet winner $(\mathrm{CW})$ if for any other outcome $y, x$ gives a higher payoff than $y$ to a majority of voters).

\section{The experiments}

Our experimental design is built on the main motivation of the paper, that is, studying the behaviour of voters in simultaneous and iterative voting for multiple referenda and, in particular, investigating the impact of iterative voting on individual and collective behavior. Below we summarize the experimental procedure that we implemented.

\subsection{Experimental Design}

In our experimental design, each participant took the role of a voter in nine elections. The first four of these elections were implemented as oneshot voting, in which subjects are told that they are in a group of 7 and will make choices for columns and rows based on their individual payoff matrices, without any further information. The collective choice for both the column and the row are reached with the majority rule to determine the final outcome.

In the remaining five elections, which were implemented as iterated voting, groups consisted of either 3,5 , or 7 voters. $^{8}$ After each stage within an election, subjects are provided with the vote tally for each column and row and the final outcome in all previous stages. The final outcome of an iterated election was reached if an outcome is repeated for three stages. In case this is not achieved, the outcome at 12th stage is assigned as the final outcome. Subjects and groups are randomly assigned to voter profiles at each election. The elections were based on the set of profiles we introduce in what follows.

\footnotetext{
${ }^{8}$ In elections with 5 voters (Profiles 8 and 9), one subject among the 21 present in the lab is randomly chosen to wait through the election.
} 


\subsubsection{Profiles in the experiment}

In Profile 1, which is voted one-shot, all preferences are fully separable. $B R$ is the Condorcet winner. The average social welfare for each outcome is described in Table 8 for all profiles.

\begin{tabular}{|c|c|c|c|c|c|c|c|c|c|c|c|}
\hline \multicolumn{3}{|c|}{$\frac{\text { Voters } 1 \& 2}{\mathrm{~L} \quad \mathrm{R}}$} & \multicolumn{3}{|c|}{$\frac{\text { Voters } 3 \& 4}{\mathrm{~L} \quad \mathrm{R}}$} & \multicolumn{3}{|c|}{$\frac{\text { Voters } 5 \& 6}{\mathrm{~L} \quad \mathrm{R}}$} & \multicolumn{3}{|c|}{$\frac{\text { Voter 7: }}{\mathrm{L}} \mathrm{R}$} \\
\hline . & 2 & 3 & $\mathrm{~T}$ & 1 & 0 & $\mathrm{~T}$ & 0 & 2 & $\mathrm{~T}$ & 3 & 1 \\
\hline B & 0 & 1 & B & 3 & 2 & B & 1 & 3 & B & 2 & 0 \\
\hline
\end{tabular}

Table 1: Profile 1.

In Profiles 2 to 4, which are voted one-shot, no preference is separable in any issue. In all cases, $B L$ is the Condorcet winner.

\begin{tabular}{|c|c|c|c|c|c|c|c|c|c|c|c|}
\hline \multicolumn{3}{|c|}{$\frac{\text { Voters } 1 \& 2}{\mathrm{~L} \quad \mathrm{R}}$} & \multicolumn{3}{|c|}{ Voters $3 \& 4$} & \multicolumn{3}{|c|}{ Voters $5 \& 6$ : } & \multicolumn{3}{|c|}{ Voter 7: } \\
\hline $\mathrm{T}$ & $\mathrm{b}$ & $\mathrm{d}$ & $\mathrm{T}$ & $\mathrm{b}$ & $\mathrm{c}$ & $\mathrm{T}$ & $\mathrm{c}$ & $\mathrm{a}$ & $\mathrm{T}$ & $\mathrm{c}$ & $\mathrm{b}$ \\
\hline B & $\mathrm{c}$ & $\mathrm{a}$ & B & $\mathrm{d}$ & $\mathrm{a}$ & B & $\mathrm{b}$ & $\mathrm{d}$ & B & $\mathrm{a}$ & $\mathrm{d}$ \\
\hline
\end{tabular}

Table 2: Profiles 2,3, and 4, where for Profile 2 the utility vector $(a, b, c, d)=$ $(0,2,3,4)$ for Profile $3:(a, b, c, d)=(0,1,2,3)$ and for Profile $4(a, b, c, d)=$ $(0,1,2,4)$

In Profile 5, which is voted in iteration, voter 3 has a fully separable preference. Voters 1 and 2 have semi-separable preferences. $B R$ is the Condorcet winner.

\begin{tabular}{|c|c|c|c|c|c|c|c|c|}
\hline \multicolumn{3}{|c|}{ Voter 1: } & \multicolumn{3}{|c|}{$\underline{\text { Voter 2: }}$} & \multirow{2}{*}{\multicolumn{3}{|c|}{$\frac{\text { Voter 3: }}{L}$}} \\
\hline & $\mathrm{L}$ & $\mathrm{R}$ & & $\mathrm{L}$ & $\mathrm{R}$ & & & \\
\hline$T$ & 1 & 0 & $\mathrm{~T}$ & 2 & 0 & $\mathrm{~T}$ & 1 & 3 \\
\hline B & 1 & 2 & B & 1 & 1 & B & 0 & 2 \\
\hline
\end{tabular}

Table 3: Profile 5.

In Profile 6, which is voted in iteration, no voter has a fully separable preference. Voters 1 and 2 have a separable preference in row issue, whereas voters 3,4 , and 7 have separable preferences in column issue. $T L$ is the Condorcet winner.

In Profile 7 , which is voted in iteration, only voter 7 has a (fully) separable preference. $T R$ is the Condorcet winner. 


\begin{tabular}{|c|c|c|c|c|c|c|c|c|c|c|c|}
\hline & $\frac{\text { ers }}{L}$ & $\frac{\& 2}{R}$ & & $\frac{\mathrm{ers}}{\mathrm{L}}$ & $\frac{\& 4}{R}$ & & $\frac{\mathrm{ers}}{\mathrm{L}}$ & $\frac{\&}{R}$ & & $\frac{\mathrm{er}}{\mathrm{L}}$ & $\mathrm{R}$ \\
\hline $\mathrm{T}$ & 3 & 4 & $\mathrm{~T}$ & 3 & 2 & $\mathrm{~T}$ & 2 & 1 & $\mathrm{~T}$ & 2 & 1 \\
\hline B & 2 & 0 & B & 4 & 0 & B & 0 & 3 & B & 3 & 0 \\
\hline
\end{tabular}

Table 4: Profile 6.

\begin{tabular}{|c|c|c|c|c|c|c|c|c|}
\hline \multicolumn{3}{|c|}{ Voters $1-3$ : } & \multicolumn{3}{|c|}{ Voters 4-6: } & \multicolumn{3}{|c|}{ Voter 7: } \\
\hline & $\mathrm{L}$ & $\mathrm{R}$ & & $\mathrm{L}$ & $\mathrm{R}$ & & $\mathrm{L}$ & $\mathrm{R}$ \\
\hline $\mathrm{T}$ & 0 & 3 & $\mathrm{~T}$ & 0 & 4 & $\mathrm{~T}$ & 0 & 2 \\
\hline B & 4 & 1 & B & 3 & 1 & B & 1 & 3 \\
\hline
\end{tabular}

Table 5: Profile 7.

In Profile 8, which is voted in iteration, voters 1,2 , and 4 have fully separable preferences, whereas voter 5 has a separable preference only in the column issue. $T L$ is the Condorcet winner.

\begin{tabular}{|c|c|c|c|c|c|c|c|c|c|c|c|c|c|c|}
\hline \multicolumn{3}{|c|}{$\frac{\text { Voter 1: }}{\mathrm{L}} \mathrm{R}$} & \multicolumn{3}{|c|}{$\frac{\text { Voter 2: }}{\mathrm{L}} \mathrm{R}$} & \multicolumn{3}{|c|}{$\frac{\text { Voter } 3:}{\mathrm{L}} \mathrm{R}$} & \multicolumn{3}{|c|}{$\frac{\text { Voter 4: }}{\mathrm{L}} \mathrm{R}$} & \multicolumn{3}{|c|}{$\frac{\text { Voter 5: }}{\mathrm{L}} \mathrm{R}$} \\
\hline & 3 & 2 & $\mathrm{~T}$ & 2 & 3 & $\mathrm{~T}$ & 2 & 0 & $\mathrm{~T}$ & 1 & 0 & $\mathrm{~T}$ & 2 & 1 \\
\hline & 1 & 0 & B & 0 & 1 & B & 1 & 3 & B & 3 & 2 & B & 3 & 0 \\
\hline
\end{tabular}

Table 6: Profile 8.

In Profile 9, which is voted in iteration, only the voter 2 has a fully separable preference. Voters 4 and 5 have separable preferences only in the column issue. The pairwise majority relation is cyclic, hence there is no Condorcet winner.

\subsubsection{Overview of the experimental profiles}

Profile 1 allows us to look into the tendency to vote rationally, avoiding dominated choices. Profiles 2 to 4 place voters in a dilemma between optimism and pessimism for one issue, while the other issue pertains to SD-rationality. Also, comparing the individual behaviour within these profiles allow us to investigate the behavioral effects of the convexity of the utilities.

In Profile 5, voters need to get away from a bad outcome by coordinating. If voter 3 is rational, and if voters 1 and 2 are both SD-rational, and either both optimistic or both pessimistic, then the outcome is $T R$ or $B L$, which is respectively the worst outcome for both, and the second worst outcome for both, while $B R$ or $T L$ are Pareto-optimal for them.

In Profile 6, $T L$ represents a good compromise (in particular, it is both a 


\begin{tabular}{|c|c|c|c|c|c|c|c|c|c|c|c|c|c|c|}
\hline \multicolumn{3}{|c|}{ Voter 1: } & \multicolumn{3}{|c|}{$\frac{\text { Voter 2: }}{\mathrm{L}}$} & \multicolumn{3}{|c|}{ Voter 3: } & \multicolumn{3}{|c|}{ Voter 4: } & \multicolumn{3}{|c|}{ Voter 5: } \\
\hline & 1 & 3 & $\mathrm{~T}$ & 2 & 0 & $\mathrm{~T}$ & 2 & 1 & $\mathrm{~T}$ & 0 & 3 & $\mathrm{~T}$ & 3 & 0 \\
\hline & 2 & 0 & B & 3 & 1 & B & 0 & 3 & $\mathrm{~B}$ & 1 & 2 & B & 2 & 1 \\
\hline
\end{tabular}

Table 7: Profile 9.

\begin{tabular}{r|c|c|c|c} 
Profile & $B L$ & $T L$ & $T R$ & $B R$ \\
\hline 1 & $11 / 7$ & $9 / 7$ & $12 / 7$ & $10 / 7$ \\
2 & $18 / 7$ & $17 / 7$ & $16 / 7$ & $12 / 7$ \\
3 & $9 / 7$ & $10 / 7$ & $11 / 7$ & $12 / 7$ \\
4 & $14 / 7$ & $10 / 7$ & $13 / 7$ & $16 / 7$ \\
5 & $2 / 3$ & $4 / 3$ & $3 / 3$ & $5 / 3$ \\
6 & $15 / 7$ & $18 / 7$ & $15 / 7$ & $6 / 7$ \\
7 & $22 / 7$ & 0 & $23 / 7$ & $9 / 7$ \\
8 & $8 / 5$ & 2 & $6 / 5$ & $6 / 5$ \\
9 & $8 / 5$ & $8 / 5$ & $7 / 5$ & $7 / 5$
\end{tabular}

Table 8: Social welfare of each outcome for all the considered profiles.

Condorcet winner and a social welfare optimal), but it may not be reached at the first stage. Since the profile is rather consensual and not 'pathological', one would expect a rather rapid convergence to $T L$.

Profile 7 is an example of profile where the outcome at the first stage is expected to be very bad: assuming that a large enough part of voters 1-6 vote SD-rationally, the outcome would occur to be $B R$, which gives utility 1 to all: for these six voters, $B R$ is Pareto-dominated by $B L$ and $T R$ but they must in some way coordinate to elect one of them, which may take several steps. ${ }^{9}$

Profile 8 is different enough from the previous profiles because three voters out of five have separable preferences and are therefore expected not to change their votes, and voter 5 has a dominant action $(L)$; therefore the outcome would be expected to be $B L$ or $T L$ (the CW). Finally, in Profile 9 the pairwise majority relation is cyclic and two outcomes yield the same (highest) level of average social welfare.

\subsection{Experimental Procedure}

We recruited 147 subjects that participated in groups of 21 in 7 sessions or-

\footnotetext{
${ }^{9}$ Another interest of Profile 7 is that if the current outcome is $B R$, and if the margins of victory for both $B$ and $R$ are large, then voters 1-6 would feel that their probability of being pivotal at the next stage is low for each of both issues, and may be tempted to vote $T L$ to try to be pivotal in either one them (the probability of being pivotal on both being negligible); such a behaviour has been analyzed theoretically by Ahn and Oliveros (2012).
} 
ganized at the Laboratoire d'Economie Experimentale de Paris in September 2019. Only one third of our subjects were students. When they arrived in the lab, subjects were randomly assigned to a computer terminal. General instructions were read aloud and distributed in print at the terminals. These instructions can be found in the Appendix B. Subjects were informed that the experiment consisted of a set of nine elections. It is made explicit that subjects would be paid in cash at the end of the experiment (with conversion rate of 1 experimental point $=1$ Euro), based on their and the other participants' choices in elections. Questions are taken before the start of the rounds and no questions were allowed during the experiment. The experiments started following specific instructions about how to read the screens. The experiments were computerized with jtree (Powell, 2019) and we are grateful to the support we received from Owen Powell.

Before each election round, it is specified if the election will be implemented as one-shot or in iteration. In elections with iteration, i.e., profiles 5 to 9 , it is explained that the elections would conclude if the outcome repeats for three rounds. In case three rounds of repetition did not occur, the election would end at the 12th round.

At the end of the experiment, we conducted a short questionnaire on demographics and subjects' perceptions about iterated elections, which can be found in the Appendix. Average payment per subject was 16 Euros (ranging from 4 to 26). In addition to that, we paid 5 Euros participation fee to every subject, including those who arrived but could not participate as all the slots were filled, as well. On average, the whole experiment from instructions to the payments lasted 45 minutes.

\section{Rationalizability and optimistic behavior}

In this section we focus on the individual behavior of subjects with regard to rationalizability and optimism vs. pessimism. We focus mostly on choices made in the one-shot votes and first stages of iterative votes, as learning in regard to others' preferences is not an issue in these cases. We first look at the prevalence of deviations from rationality, taken as choosing a dominated action. Then, we look at the SD-rationalizability in the case of fully nonseparable preferences. Finally, we look at the prevalence of optimism and pessimism in the cases of fully non-separable and semi-separable preferences.

\subsection{Dominated choices}

Hypothesis 1 Voters avoid dominated choices.

In Table 9 we present the number of instances (pairs of voter and profile) in which subjects made a dominated choice on both issues (given separable preferences), a dominated choice on one issue having separable preferences, 
and a dominated choice on one issue having semi-separable preferences. We also present in Table 10 the number of voters who made at least one dominated choice in any of the profiles considered, on both issues or on at least one issue (without separating separable or semi-separable preferences).

\begin{tabular}{r|cc} 
& Profile 1 & Profiles 5-9 \\
\hline Dominated choice in both issues & $11(7.5 \%)$ & $8(4 \%)$ \\
Dominated choice in one issue (separable pref.) & $26(17.7 \%)$ & $36(12.8 \%)$ \\
Dominated choice in one issue (semi-sep. pref.) & - & $47(16.4 \%)$
\end{tabular}

Table 9: The number (percentage) of dominated choices in one-shot elections and first stages of the iterative elections. The percentages are calculated on the total number of potential dominated choices: 147 for Profile 1, 182 for Profiles 5-9 for separable preferences, and 287 for semi-separable preferences.

\begin{tabular}{r|c} 
& Profiles 1 \& 5-9 \\
\hline At least one dominated choice in both issues & $16(11 \%)$ \\
At least one dominated choice in at least one issue & $61(41 \%)$
\end{tabular}

Table 10: The number (percentage) of subjects who made a dominated choice at least once in one-shot elections or first stages of the iterative elections.

Our findings show that violation of rationality is not prevalent in oneshot voting, save for $25.5 \%$ of instances in which deviation from rationality is observed, supporting Hypothesis 1. No statistically significant difference is detected comparing one-shot and (first stages of) iterative elections (Fisher's exact tests yield $p>0.1$ ). Thus, we conclude that the presence of iteration does not have an impact on the rationality of choices. We also did not observe a statistically significant difference between the proportion of subjects with fully separable preferences who made dominated choices and the proportion of subjects with semi-separable preferences who made dominated choices $\left(p>0.1\right.$ in Fisher's test). ${ }^{10}$

\subsection{SD-rationalizable choices}

Hypothesis 2 Voters choose SD-rationalizable actions.

Recall that a voter with fully non-separable preferences necessarily has an issue for which one choice is SD-rationalizable. Here, we focus on the frequency of SD-rationalizable choices by subjects.

\footnotetext{
${ }^{10}$ Note that this finding is based only on first stages of profiles 5 to 9 , since there are no voters with semi-separable preferences in profiles 1-4.
} 
As Table 11 below shows, both in the one-shot elections and in the first stages of iterated elections, most of the subjects with fully non-separable preferences made SD-rationalizable choices, in support of Hypothesis 2.

\begin{tabular}{ccccccc} 
Profile 2 & Profile 3 & Profile 4 & Profile 6 & Profile 7 & Profile 8 & Profile 9 \\
\hline $70.7 \%$ & $78.2 \%$ & $79.6 \%$ & $78.6 \%$ & $80.2 \%$ & $78.6 \%$ & $75.0 \%$ \\
$(147)$ & $(147)$ & $(147)$ & $(42)$ & $(126)$ & $(28)$ & $(56)$
\end{tabular}

Table 11: The percentage of subjects who made SD-rationalizable choices in the one-shot elections (Profiles $2-4$ ) and first stages of iterated elections including voters with fully non-separable preferences. The number of all subjects who had fully non-separable preferences are in parentheses.

\subsection{Optimistic/pessimistic choices}

Recall that when a voter has a semi-separable or fully non-separable preference, she has necessarily an issue for which the two choices can be characterized exclusively as optimistic and pessimistic. Here we focus on the frequency of optimistic choices in these cases.

Hypothesis 3 Voters choose optimistic actions more often than pessimistic actions).

Hypothesis 4 Voters choose optimistic actions more often when payoffs are more convex.

Hypothesis 5 Voters choose optimistic actions more often in iterative elections than in one-shot elections.

Table 12 below shows the percentage of optimistic choices in one-shot elections, whereas Table 13 shows the corresponding values for the first stages of iterative elections. In both cases, we can conclude that a majority of subjects (between $63 \%$ and $82 \%$ ) make optimistic choices, in support of Hypothesis 3. This finding confirms a phenomenon that has been observed informally by some of us for many years. Of course we have to be cautious about the psychological interpretation of "optimistic" and "pessimistic": subjects are not necessarily aware that they behave optimistically or pessimistically, but, at least, they behave as if they were optimistic or pessimistic.

Profiles 2, 3, and 4 are played one-shot and feature voters who have the same ordering over the outcomes but different utility scales. In Profile 2 , the utility vector is $(0,2,3,4)$, in Profile 3 , it is $(0,1,2,3)$, whereas in Profile 4 , it is $(0,1,2,4)$. One can argue that subjects would avoid the worst alternative more often in Profile 2 than in Profile 3 (hence tend to be more pessimistic), since this brings about a larger relative loss. Similarly, subjects would be 
attracted more by the best alternative in Profile 4 since it leads to greater relative gain (hence tend to be more optimistic, also as the small span action pays less). We observe that choices are significantly more optimistic going from Profile 2 to 3 and/or to Profile 4, supporting Hypothesis 4. The Fisher exact test statistic values are 0.624 for comparing Profiles 2 and 3, 0.077 for comparing Profiles 2 and 4, and 0.248 for comparing Profiles 3 and 4.

\begin{tabular}{ccc} 
Profile 2 & Profile 3 & Profile 4 \\
\hline $63.9 \%$ & $67.3 \%$ & $74.1 \%$
\end{tabular}

Table 12: The percentage of optimistic choices by profiles with different utility scales. In all cases, $n=147$.

\begin{tabular}{ccccc} 
Profile 5 & Profile 6 & Profile 7 & Profile 8 & Profile 9 \\
\hline $71.4 \%$ & $76.2 \%$ & $73.8 \%$ & $82.1 \%$ & $75.0 \%$ \\
$(98)$ & $(147)$ & $(126)$ & $(56)$ & $(112)$
\end{tabular}

Table 13: The percentage of optimistic choices in the first stages of iterative elections. The number of subjects are in parentheses.

Next, we look whether the percentage of observed optimistic choices depend on the presence of iteration. Thus, in what follows, we compare choices in one-shot elections and the first stages in iterative elections. However, we need to focus on comparable cases, i.e., all voters in Profile 3 and voters 5 and 6 in Profile 6, voter 3 in Profile 8 and voters 1 and 3 in Profile 9. ${ }^{11}$ These voters have fully non-separable preferences and utilities are of the form 3210. We find that there is no statistically significant difference between the one-shot profiles and the first stages of iterated profiles in terms of the observed percentages of optimistic choices (differences between Profile 3 and others are not significant at $10 \%$ according to Fisher's exact test, see Table 14), indicating no statistical support for the argument that iteration leads to more optimistic choices. Hypothesis 5, thus, does not find support in our experimental data.

\section{Individual Voting Dynamics}

In this section we focus on iterative elections and investigate how subjects' choices evolve in relation to the separability of their preferences and to the outcomes and their pivotality in previous rounds.

\footnotetext{
${ }^{11}$ The choices in Profiles 2 and 4 cannot be compared with choices by any other voter in an iterated profile.
} 


\begin{tabular}{cccc} 
Profile 3 & Profile 6 & Profile 8 & Profile 9 \\
\hline $67.3 \%$ & $78.6 \%$ & $78.6 \%$ & $69.6 \%$
\end{tabular}

(147)

Table 14: The percentage of optimistic choices in one-shot elections (Profile 3 ) and first stages of iterative elections (Profiles 6, 8, and 9) for voters with utility scale 3210 . The numbers of subjects are printed in parentheses.

Hypothesis 6 Voters with non-separable preferences change their choices more often than voters with separable preferences.

\begin{tabular}{r|rcc|rcc} 
& \multicolumn{4}{|c}{ Separable } & \multicolumn{3}{c}{ Non-separable } \\
Profile & Voters & No Change & Any Change & Voters & No Change & Any Change \\
\hline 5 & 3 & 25 & 24 & - & - & - \\
6 & - & - & - & 5,6 & 16 & 26 \\
7 & 6 & 15 & 6 & $1-6$ & 54 & 72 \\
8 & $1,2,4$ & 44 & 40 & 3 & 13 & 15 \\
9 & 2 & 12 & 16 & 1,3 & 21 & 35 \\
\hline All & & 96 & 86 & & 104 & 148
\end{tabular}

Table 15: Number of subjects with separable and non-separable preferences who change their votes at least once and who do not change at all.

As a first level analysis, we compare the number of subjects who change their votes at least once and those who never change, based on whether their preferences are separable or not, given in Table 15. According to Fisher's exact test, the difference is statistically significant (comparing 148 out of 252 and 86 out of 182 , test statistic value is 0.019 ) at $5 \%$, hence we indeed observe a higher tendency to change when preferences are non-separable, in the line of Hypothesis 6.

We next estimated the following linear probability model to detect the effect of separability on the vote changing behavior:

$$
\Delta x_{i t}=\beta_{0}+\beta_{1} S E P_{i t}+u_{i t},
$$

where $\Delta x_{i t}$ equals 1 if voter $i$ changed her vote for either of the issues from stage $t-1$ to $t$ and 0 otherwise, $S E P_{i t}$ equals 1 if the preference is separable and 0 if non-separable, and $u_{i t}$ denote the error term. ${ }^{12}$ As can be seen in the Table 16 below, which shows the estimation results where standard errors are clustered at the subject level, although the effect is not large (about $7 \%$ higher probability of changing a vote in case of non-separable preferences

\footnotetext{
${ }^{12}$ The estimation is restricted to fully separable and fully non-separable preferences. Semi-separable cases are analyzed in the sequel.
} 
compared to separable preferences), we observe that non-separability leads to more change.

\begin{tabular}{lcccc} 
& Estimate & Std. Error & $t$ statistic & $p$-value \\
\hline Intercept & 0.324 & 0.027 & 12.156 & 0.000 \\
SEP & -0.069 & 0.038 & -1.830 & 0.067
\end{tabular}

Table 16: OLS estimation results for the model (1). Standard errors are clustered at subject level. $n=1607$. Two-tailed $p$-values are reported.

Table 17 presents vote changing behavior when preferences are semi-separable. We see that subjects are more likely to change their votes for the nonseparable issues (Fisher exact test statistic value is 0.006 comparing 98 vote changes in separable issues and 131 changes in non-separable issues out of 287), in support of Hypothesis 6.

\begin{tabular}{r|r|cc|cc} 
& & \multicolumn{2}{|c}{ Separable issue } & \multicolumn{2}{c}{ Non-separable issue } \\
Profile & Voters & No Change & Any Change & No Change & Any Change \\
\hline 5 & $1-2$ & 53 & 45 & 52 & 46 \\
6 & $1-4,7$ & 72 & 33 & 57 & 48 \\
8 & 5 & 22 & 6 & 19 & 9 \\
9 & 4,5 & 42 & 14 & 28 & 28 \\
\hline All & & 189 & 98 & 156 & 131
\end{tabular}

Table 17: Number of subjects with semi-separable preferences who change and who do not change their votes across profiles.

We now look at how subjects respond to the outcomes of previous rounds. We assume that subjects would want to improve the outcome for themselves. We say that a voter is pivotal when the outcome of the previous round has a margin of victory of one on any of the two issues. Thus, provided that all other voters keep the same vote, the voter can switch the outcome by unilateral deviation.

Hypothesis 7 Voters change their votes more often if they were pivotal in the previous round.

Table 18 below shows the number of subjects who change their votes from round $t-1$ to $t$, based on if they were pivotal in round $t-1$, given that they did not vote for their optimal choice in round $t-1$ and that their optimal choice was not chosen. We observe no statistically significant difference (Fisher's exact test yields $p>0.1$ ).

Table 19 below shows the number of subjects who change their votes from round $t-1$ to $t$, based on if they were pivotal in round $t-1$, given 


\begin{tabular}{rcc} 
& No Change & Any Change \\
\hline Pivotal in the previous round & $299(56.9 \%)$ & 226 \\
Not pivotal in the previous round & $175(58.7 \%)$ & 123
\end{tabular}

Table 18: The number of subjects who change their votes by pivotality in the last round. Only those subjects who did not vote for their optimal choice in last round and that this was not chosen are included. Percentages are printed in parentheses.

that the outcome of last round was the worst outcome for themselves. We observe that pivotality has a significant impact in these cases, as the Fisher exact test statistic value is $0.020(p$-value $<.05) .{ }^{13}$ In conclusion, we find a partial support for Hypothesis 7: subjects pay attention to pivotality only in case they get a bad outcome.

\begin{tabular}{rcc} 
& No Change & Any Change \\
\hline Pivotal in the previous round & $124(48.1 \%)$ & 134 \\
Not pivotal in the previous round & $83(37.0 \%)$ & 140
\end{tabular}

Table 19: The number of subjects who change their votes by pivotality in the last round. Only those instances where in the last round the worst outcome for the subject was chosen are included. Percentages are printed in parentheses.

We also calculated the percentage of best responses (i.e., vote for their favourite cell) among the vote changes induced by pivotality (see Table 20), finding that in a majority of the cases subjects best respond when pivotal on both issues. ${ }^{14}$

\footnotetext{
${ }^{13}$ When the outcome of the last period is not the worst, $27.0 \%$ of the (1239) times the subjects change their votes when they were pivotal, compared to $30.7 \%$ (out of 661 cases) when they were not pivotal.

${ }^{14}$ We have also checked whether voters with fully nonseparable preferences would vote for the opposite of their favourite cells, when the perceived probability of being pivotal on both issues is very low, in line with the analysis by Ahn and Oliveros (2012). Instances of such a phenomenon occur in the following circumstances, (1) fully non separable preferences, such as voters 1-6 in Profile 7; 2 the current outcome is the second worst outcome ( $B R$ for these 6 voters); (3) the margin of victory for both issues is large (e.g., without counting the voter, $5-1$ or $6-0)$; (4) at the next round, the voter changes their vote on both issues ( $T L$ for the latter 6 voters). However, we cannot say much: among four occurrences of (1) and (2), there are two that comply with our argument. This could be worth line of inquiry to pursue in future research with an experimental design that is aimed to focus on this phenomenon.
} 


\begin{tabular}{ccccc} 
Profile 5 & Profile 6 & Profile 7 & Profile 8 & Profile 9 \\
\hline $62 \%$ & $55 \%$ & $71 \%$ & $64 \%$ & $70 \%$
\end{tabular}

Table 20: The percentage of best-response choices when subjects are pivotal on both issues.

\section{Collective dynamics and quality of the final out- come}

In this section we analyze the collective behavior of the voters in the iterated elections (i.e., Profiles 5 to 9) and evaluate the election outcomes in terms of social welfare and the frequency of electing a Condorcet winner.

Recall that the termination rule puts an end to the iteration when the outcome does not change for three rounds. As can be seen in Figure 2 many groups concluded their iterative voting process at the end of the third stage, the earliest possible. Only $53,29,43,36$, and $46 \%$ of groups remain in iteration beyond the third stage in profiles 5 to 9 respectively. All groups in Profile 7 conclude by stage 6 .

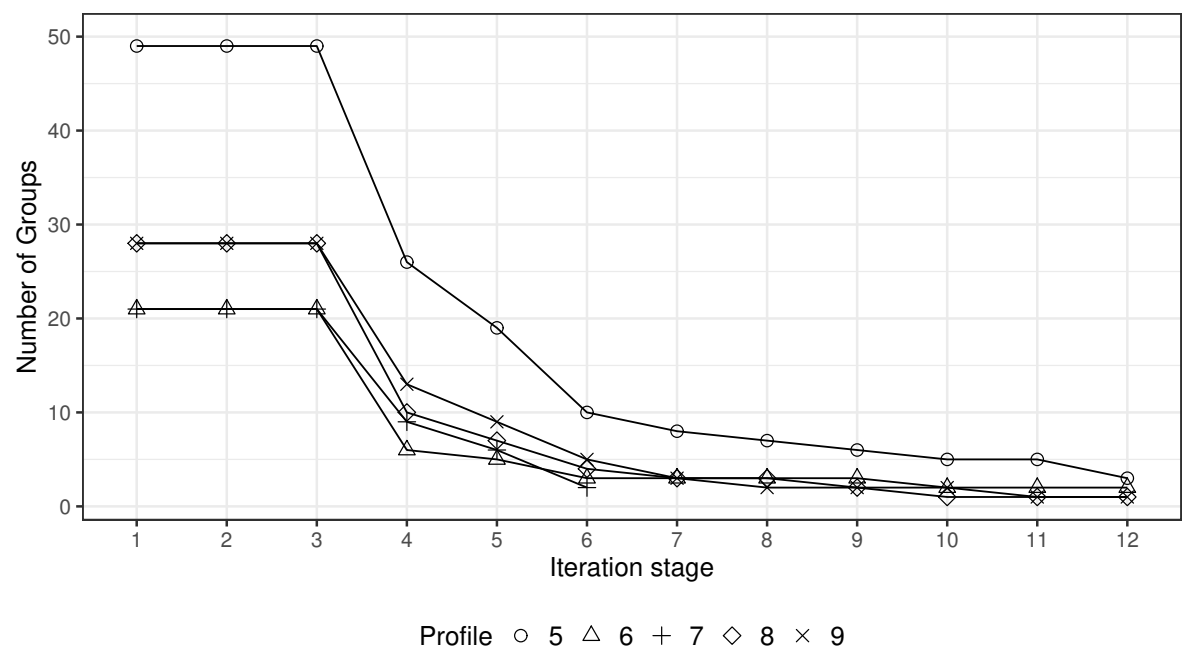

Figure 2: Number of groups in the iterated votes by iteration stage.

We divide the groups of voters into two categories in terms of the evolution of election outcomes. The static are those groups who terminate at the third iteration stage, and the dynamic groups are those who go further. We observe that for both types of groups there are significant numbers of subjects who change their votes. More precisely, the percentage of subjects in dynamic groups who update their vote in the first three rounds is in the 
range of $20-49 \%$, and the same figure is in the range of $13-36 \%$ in static groups, depending on the different profiles played and the iteration stage (see Table 25 in Appendix A).

We next look at two measures to evaluate the quality of the election outcomes, in line with previous work in iterative voting, namely, the average social welfare and the frequency of the election of Condorcet winners (Reijngoud and Endriss, 2012; Grandi et al., 2013; Wilczynski, 2019; Meir et al., 2020).

Hypothesis 8 Iterative voting increases average social welfare.

We measure the social welfare as the average utility of the election outcome within groups. ${ }^{15}$ Table 21 shows the average social welfare in the first and last stage of iteration for Profiles 5-9, in both aggregated form and divided between dynamic and static groups. The maximum and minimum possible values for the average social welfare are also included.

\begin{tabular}{r|cc|cc|cc|c} 
& & & \multicolumn{2}{|c}{ All } & \multicolumn{2}{c}{ Dynamic } & Static \\
Profile & Max & Min & First & Last & First & Last & First = Last \\
\hline 5 & 1.67 & 0.67 & 1.27 & 1.39 & 1.15 & 1.37 & 1.42 \\
6 & 2.57 & 0.86 & 1.88 & 1.99 & 1.58 & 1.98 & 1.99 \\
7 & 3.29 & 0.00 & 2.21 & 2.65 & 1.74 & 2.77 & 2.57 \\
8 & 2.00 & 1.20 & 1.59 & 1.66 & 1.6 & 1.8 & 1.58 \\
9 & 1.60 & 1.40 & 1.52 & 1.52 & 1.54 & 1.54 & 1.51
\end{tabular}

Table 21: Average social welfare in the first and last iteration stage. The column "All" includes all groups, "dynamic" include only groups that went beyond third stage, and the column "static" includes groups that concluded in the first three periods. The first column indicates the highest and lowest average social welfare attainable in each profile.

We observe that iterative voting significantly increases average social welfare in all profiles, which confirms the conclusion of the simulations in Bowman et al. (2014) and our Hypothesis 8. The average last stage social welfare for dynamic groups in all profiles is 1.72 , whereas average first stage social welfare is 1.42 . Wilcoxon signed-rank (paired) test yields a $p$-value of 0.000 in support of the alternative hypothesis that last stage average social welfare is higher (in other words, the last stage average social welfare is statistically significantly higher than the first stage average social welfare).

In Profiles 5 to 7 , the static groups started with an election outcome with a higher social welfare than the one of the dynamic groups, who eventually

\footnotetext{
${ }^{15}$ Note that this slightly contrasts with the measure used by Bowman et al. (2014), who computes the social welfare as the Borda score of the election outcome divided by the number of voters. The two measures differ only on profiles with utility scales that are not linear.
} 
improve it to a comparable level in the last stage of iteration. ${ }^{16}$

Interestingly, these results contrast with the findings of Meir et al. (2020) in online iterated elections, who do not find any statistically significant improvement in social welfare through iteration. This suggests that multi-issue elections are more likely to profit from an iterative voting scheme than classical plurality elections over a set of candidates.

We then investigated the impact of iterative voting on the Condorcet efficiency, i.e., the probability of electing a Condorcet winner when there is one. In all our profiles but the last one (9) there is a Condorcet winner. Table 22 below shows the frequency of Condorcet winners being chosen in one-shot votes (Profiles 1-4) and Table 23 those in the first stage of iterated elections (Profiles 5-8). The observed level of Condorcet efficiency is quite low. However, it is not completely clear what conclusions can be drawn from these numbers, as most of our profiles were chosen so as to be at least somewhat pathological in the first place and not fit for this particular question.

\begin{tabular}{r|c} 
Profile & Frequency of chosen CWs \\
\hline 1 & $24 \%$ \\
2 & $19 \%$ \\
3 & $24 \%$ \\
4 & $5 \%$
\end{tabular}

Table 22: Frequency of chosen Condorcet winners by profile in one-shot votes.

\begin{tabular}{r|cc|cc|c} 
& \multicolumn{2}{|c}{ All } & \multicolumn{2}{c}{ Dynamic } & Static \\
Profile & First & Last & First & Last & First = Last \\
\hline 5 & $35 \%$ & $49 \%$ & $19 \%$ & $46 \%$ & $52 \%$ \\
6 & $24 \%$ & $24 \%$ & $17 \%$ & $17 \%$ & $27 \%$ \\
7 & $52 \%$ & $29 \%$ & $78 \%$ & $22 \%$ & $33 \%$ \\
8 & $29 \%$ & $18 \%$ & $30 \%$ & $0 \%$ & $28 \%$
\end{tabular}

Table 23: Frequency of election of the Condorcet winner in iterated profiles in the first and last iteration. The column "All" includes all groups, "dynamic" only those groups going beyond three iterations, and "static" those who stopped after three iterations.

As for the effects of iteration on Condorcet efficiency, there is no clear indication of increase or decrease. In all profiles played in iteration, the Condorcet winner is also the social welfare optimum; this is not a guarantee

\footnotetext{
${ }^{16}$ Note that Profiles 8 and 9 do not have a very large span of social welfare between the worst and best outcome.
} 
of this outcome to be elected: in Profile 8, TL is elected in less than $50 \%$ of the cases. Given our findings, the observed increase in social welfare is due more to voters avoiding bad outcomes than improving good ones. This is corroborated by our results on pivotality that show that voters tend to change their vote only if they get a particularly bad outcome.

\section{Conclusions}

Our experiments allowed us to verify some of our hypotheses, and to confirm another one that had been verified only in simulations. In summary:

- in presence of separability, subjects tend to vote rationally.

- in presence of non-separability, subjects tend to play a stochastically dominant action when they have one, and otherwise tend to vote optimistically more often than pessimistically; the frequency of optimism increases with the convexity of payoffs (but does not increase with time in iterated voting).

- in iterated elections subjects with non-separable preferences tend to change their choices more often than voters with separable preferences.

- in iterated elections subjects tend to change their votes more often if they were pivotal in the previous round, provided they got a bad outcome in that round (in other cases there is no evidence that pivotality has an influence on changing votes).

- iterative voting increases social welfare, which confirms the findings by Bowman et al. (2014), obtained from simulations. On the other hand, our experiments do not allow us to draw any conclusion about whether iterative voting increases Condorcet-efficiency.

An important caveat of our results is the restriction to two (binary) issues. This choice was clearly motivated by feasibility: implementing an experiment with three issues or more makes it difficult to write the payoff matrices in a user-friendly way, the experiment would have been more cognitively complex and the risk would then have been that a significant number of voters do not understand it well or that they find it too demanding and end up playing randomly. As a consequence, it makes it difficult to compare directly with the simulation results in Bowman et al. (2014), who considered three binary issues.

This leads us to the following important question: which of our conclusions would still hold for more than two issues? For some of them, while it is impossible to have a guarantee that they indeed would hold not having made any further experiment, it can still be reasonable to assume that they would continue to hold. This is the case for hypotheses 1, 2,6 and 8; this is 
also the case for hypotheses 3-5 but it is more difficult to define optimism and pessimism because these notions become gradual as soon as we have more than two variables. It is slightly more difficult to make conjectures for hypotheses 7 and 9, although we do not see a clear reason why they would not hold for more than two issues.

In any case, even if our results held for only two issues, they would still be meaningful: in most practical cases, voters' preferential dependencies between issues in multiple referenda can be restricted to sets of issues of size two (although we acknowledge that situations where more than two issues interact do occur, especially for funding public projects with budget constraints).

The main practical impact of our study is a confirmation that iterative voting is a viable way of handling nonseparability in multiple referenda, confirming the simulation-based results of (Bowman et al., 2014). To push further the inclusion of deliberative tools in the iterative voting protocol, in future work we will allow "cheap talk" among voters in between the iterations on each vote, testing whether this leads to a decrease in the number of iterations or if it modifies their individual voting behaviour.

\section{Acknowledgements}

We are very much indebted to Jean-François Bonnefon who was the first to have the idea of this experiment about a decade ago and who had started to design one. We also thank Jean-François Laslier for useful suggestions about the protocol. Last but not least, we thank Maxim Frolov and Owen Powell for their huge support before and during the lab sessions. We thank the participants of the Dagstuhl seminar 19381 on "Application-Oriented Computational Social Choice," as well as our colleagues at the Institut de Recherche en Informatique de Toulouse (IRIT) for participating to various pilot versions of our lab experiment. 


\section{References}

Ahn, D. S. And S. Oliveros (2012): "Combinatorial Voting," Econometrica, 80, 89-141.

Airiau, S., U. Grandi, and F. S. Perotto (2017): "Learning Agents for Iterative Voting," in Proceedings of the 5th International Conference on Algorithmic Decision Theory (ADT).

Baumeister, D., A. Selker, And A. Wilczynski (2020): "Manipulation of Opinion Polls to Influence Iterative Elections," in Proceedings of the 19th International Conference on Autonomous Agents and Multiagent Systems (AAMAS).

Benoît, J. And L. A. Kornhauser (2010): "Only a dictatorship is efficient," Games and Economic Behaviour, 70, 261-270.

Bowman, C., J. K. Hodge, And A. Yu (2014): "The Potential of Iterative Voting to Solve the Separability Problem in Referendum Elections," Theory and Decision, 77, 111-124.

Bradley, W. J., J. K. Hodge, And D. M. Kilgour (2005): "Separable discrete preferences," Mathematical Social Sciences, 49, 335-353.

Brams, S. J., D. M. Kilgour, and M. R. Sanver (2007): "A minimax procedure for electing committees," Public Choice, 401-420.

Brams, S. J., D. M. Kilgour, And W. S. Zwicker (1997): "Voting on referenda: the separability problem and possible solutions," Electoral Studies, 16, 359 - 377.

(1998): "The paradox of multiple elections," Social Choice and Welfare, 15, 211-236.

Conitzer, V., J. Lang, And L. Xia (2011): "Hypercubewise Preference Aggregation in Multi-Issue Domains," in Proceedings of the 22nd International Joint Conference on Artificial Intelligence (IJCAI).

Cornelio, C., M. S. Pini, F. Rossi, And K. B. Venable (2019): "Multiagent soft constraint aggregation via sequential voting: theoretical and experimental results," Autonomous Agents and Multi Agent Systems, 33, 159-191.

ÇUhadaroĞLu, T. AND J. Lainé (2012): "Pareto efficiency in multiple referendum," Theory and Decision, 72, 525-536.

Elkind, E., D. Grossi, E. Shapiro, and N. Talmon (2020): "Egalitarian Deliberative Decision Making," CoRR, abs/2001.08031. 
Fain, B., A. Goel, K. Munagala, and S. Sakshuwong (2017): "Sequential Deliberation for Social Choice," in Proceedings of the 13th International Conference on Web and Internet Economics (WINE).

Garg, N., V. Kamble, A. Goel, D. Marn, and K. Munagala (2019): "Iterative Local Voting for Collective Decision-making in Continuous Spaces," Journal of Artificial Intelligence Research, 64, 315-355.

Goel, A. And D. T. Lee (2016): "Towards Large-Scale Deliberative Decision-Making: Small Groups and the Importance of Triads," in Proceedings of the 2016 ACM Conference on Economics and Computation $(E C)$.

Gonzales, C., P. Perny, and S. Queiroz (2008): "Preference Aggregation with Graphical Utility Models," in Proceedings of the 23rd AAAI Conference on Artificial Intelligence (AAAI).

Grandi, U., A. Loreggia, F. Rossi, K. B. Venable, and T. Walsh (2013): "Restricted Manipulation in Iterative Voting: Condorcet Efficiency and Borda Score," in Proceeding of the 3rd International Conference on Algorithmic Decision Theory (ADT).

Hodge, J. K., M. Krines, And J. Lahr (2009): "Preseparable Extensions of Multidimensional Preferences," Order, 26, 125-147.

Lacy, D. And E. M. S. Niou (2000): "A Problem with Referendums," Journal of Theoretical Politics, 12, 5-31.

LAFFONT, J.-J. (1987): "Incentives and the allocation of public goods," in Handbook of Public Economics, Elsevier, vol. 2 of Handbook of Public Economics, chap. 10, $537-569$.

Lang, J., J. Mengin, And L. Xia (2018): "Voting on multi-issue domains with conditionally lexicographic preferences," Artificial Intelligence, 265, $18-44$.

LANG, J. AND L. XIA (2009): "Sequential composition of voting rules in multi-issue domains," Mathematical Social Sciences, 57, 304-324.

(2016): "Voting in Combinatorial Domains," in Handbook of Computational Social Choice, 197-222.

LEV, O. AND J. S. Rosenschein (2016): "Convergence of Iterative Scoring Rules," Journal of Artificial Intelligence Research, 57, 573-591.

LiEkAH, L. (2019): "Multiagent Reinforcement Learning for Iterative Voting," Master's thesis, Université Jean Monnet and Université Toulouse 1 Capitole, France. 
MeIR, R. (2017): "Iterative voting," in Trends in Computational Social Choice, ed. by U. Endriss, AI Access, 69-86.

Meir, R., K. Gal, And M. Tal (2020): "Strategic voting in the lab: compromise and leader bias behavior," Autonomous Agents and Multi Agent Systems, 34, 31.

Meir, R., M. Polukarov, J. S. Rosenschein, and N. R. Jennings (2010): "Convergence to Equilibria in Plurality Voting." in Proceedings of the Twenty-fourth conference on Artificial Intelligence (AAAI).

(2017): "Iterative voting and acyclic games," Artificial Intelligence, $252,100-122$.

Obraztsova, S., E. Markakis, M. Polukarov, Z. Rabinovich, and N. R. Jennings (2015): "On the Convergence of Iterative Voting: How Restrictive Should Restricted Dynamics Be?" in Proceedings of the 19th AAAI Conference on Artificial Intelligence (AAAI).

Özkal-Sanver, I. AND M. R. SAnver (2006): "Ensuring Pareto Optimality by Referendum Voting," Social Choice and Welfare, 27, 211-219.

Powell, O. (2019): "jtree - a javascript toolbox for running economics experiments," Github.

Reijngoud, A. And U. Endriss (2012): "Voter Response to Iterated Poll Information," in Proceedings of the 11th International Joint Conference on Autonomous Agents and Multiagent Systems (AAMAS-2012).

Wilczynski, A. (2019): "Poll-Confident Voters in Iterative Voting," in The 33rd AAAI Conference on Artificial Intelligence (AAAI). 


\section{A Appendix: Further experimental findings}

\section{Dominated choices}

In one-shot elections, 26 out of 87 female subjects (30\%) made a choice for a dominated option (either for one issue or both), whereas 9 out of 48 male subjects (19\%) made a choice for a dominated option. The difference is not statistically significant (Fisher's test statistic value is 0.2182 ). Note that 12 subjects did not reveal their gender. We do not observe a significant difference in iterative elections either (see Table 24).

\begin{tabular}{rr|cc} 
& & Profile 1 & Profiles 5-9 \\
\hline Male & Dominated choice & $9(18.8 \%)$ & $8(22.2 \%)$ \\
& Number of subjects & 48 & 36 \\
\hline Female & Dominated choice & $26(29.9 \%)$ & $21(29.6 \%)$ \\
& Number of subjects & 87 & 71
\end{tabular}

Table 24: The number (percentage) of subjects who made a dominated choice in one-shot elections and first stages of the iterative elections, by declared gender.

As for the effect of formal education in economics, 22 out of 74 subjects (30\%) who did not have a formal education in economics made a choice for a dominated option (either for one issue or both), whereas 14 out of 72 subjects $(19 \%)$ who had a formal economic education made a choice for a dominated option. The difference is not statistically significant at $5 \%$ level as the Fisher exact test statistic value is 0.4357 .

\section{Dynamic and static groups}

Table 25 shows the percentage of subjects in each iterated profile who change their vote in the first three stages.

\begin{tabular}{r|cc|cc} 
& \multicolumn{2}{|c}{ Dynamic } & \multicolumn{2}{c}{ Static } \\
Profile & Stage 1 to 2 & Stage 2 to 3 & Stage 1 to 2 & Stage 2 to 3 \\
\hline 5 & $37 \%$ & $49 \%$ & $13 \%$ & $33 \%$ \\
6 & $31 \%$ & $40 \%$ & $29 \%$ & $30 \%$ \\
7 & $32 \%$ & $35 \%$ & $30 \%$ & $25 \%$ \\
8 & $20 \%$ & $24 \%$ & $24 \%$ & $22 \%$ \\
9 & $37 \%$ & $35 \%$ & $36 \%$ & $27 \%$
\end{tabular}

Table 25: Percentage of subjects who change their vote from 1st to $2 \mathrm{nd}$ and 2nd to $3 r d$ stages in iterated profiles. 


\section{B Appendix: Experimental Instructions}

Authors' note: In each session, the experiment consisted of 9 main parts, followed by a questionnaire and a recap of earnings. The first four parts consisted of 4 one-shot votes, whereas each of the remaining 5 parts consisted of iterated votes. The instructions, which were printed on screens as well, were read aloud in the beginning of the first and fifth parts. The following is the English translations of the original instructions in French as appeared on subjects' screens.

\section{Instructions}

Part 1

You will participate in an experiment that takes place in several parts. The experiment has 9 parts.

The first four parts consist of single turns. The following parts consist of several turns. In each game, you will see a table like this:

$\begin{array}{rcc} & \text { LEFT } & \text { RIGHT } \\ \text { TOP } & 4 & 2 \\ \text { BOTTOM } & 1 & 0\end{array}$

The number that is shown in each of the boxes is what you earn if that box wins when the game ends. For example, if the winning box when the game ends is UP-RIGHT, then for that game you earn $€ 2$. The numbers will always be at least as large as 0: you will never lose anything. Your final earning will be the sum of your earnings in the 6 games.

We will now explain what determines that a box is a winner.

You will vote for a row (UP or DOWN) and for a column (LEFT or RIGHT). In each game, there will be a number of other players who will also vote for a row and for a column. Other players may have different arrays from yours, and you cannot know the values of their arrays. However, you will know the number of players (which, depending on the case, will be 3,5 or 7$)$.

If a majority of players voted LEFT, then the winning row is LEFT. If a majority of players voted for RIGHT, then the winning row is RIGHT.

If a majority of players voted UP, then the winning column is UP. If a majority of players voted DOWN, then the winning column is DOWN.

The winning box is at the intersection of the winning row and the winning column.

If for example there are 5 players, three vote UP and two vote DOWN, then the winning row is UP. If four players vote for RIGHT and one for LEFT, then the winning column is RIGHT, and the winning box is UPRIGHT. 
During the first four elections, there will be only one round: you will vote for a row and a column, the other players too, and you will win the sum that corresponds to the winning box in the table.

\section{Part 2}

Part with only one round.

\section{Part 3}

Part with only one round.

\section{Part 4}

Part with only one round.

\section{Part 5}

The following parts will consist of several rounds. In each round you will vote for a row and a column.

Then it will be the second round: you will vote again, and at the end of the second round, again you will know the winning box and the number of votes for UP, for LOW, for LEFT, and for RIGHT. And so on, until the election ends. The election will end when the winning box is the same three times in a row or when 12 turns have been played.

For example:

- first round: UP 3, DOWN 2, LEFT 1, RIGHT 4. Winning box: UPRIGHT

- second round: UP 2, DOWN 3, LEFT 2, RIGHT 3. Winning box: DOWN-RIGHT

- third round: UP 2, DOWN 3, LEFT 3, RIGHT 2. Winning box: DOWN-LEFT

- fourth round: UP 1, DOWN 4, LEFT 2, RIGHT 3. Winning box: BOTTOM-RIGHT

- fifth round: UP 0, DOWN 5, LEFT 2, RIGHT 3. Winning box: BOTTOM-RIGHT

- sixth round: UP 1, DOWN 4, LEFT 2, RIGHT 3. Winning box: BOTTOM-RIGHT.

The DOWN-RIGHT box has won three times in a row: the game is over, and you win the amount that corresponds to the DOWN-RIGHT box.

Please note: The number of participants may vary for each part (games with 3,5 or 7 participants). When the number of participants in a game is 5 , one person in the room will not play that game. 
Please read these instructions carefully. If you have questions now is the time to ask them.

\section{Part 6}

Part with several rounds.

\section{Part 7}

Part with several rounds.

\section{Part 8}

Part with several rounds.

Part 9

Part with several rounds.

\section{Questionnaire \\ Recap of earnings}

\section{Questionnaire}

1. What is your profession?

2. Do you have any comments on the methods, or suggestions on how to organize such choices on several issues?

3. What is your age range?

- No answer

- $18-24$

- $25-34$

- $35-44$

- $45-54$

- $55-64$

- $65+$

4. You are

- No answer

- A woman

- A man

- Other

5. What is the highest degree you have obtained? 
- No answer

- Postgraduate degree, doctorate, grande école, engineer

- Graduate degree

- Undergraduate degree, BTS, DUT, or equivalent, Bac +2 level

- General, technological, professional or equivalent baccalaureate

- CAP, BEP or diploma of the same level

- College diploma, BEPC

- Primary school certificate, no diploma

6. Have you ever taken a course in economics?

- No answer

- Yes, this is my main training

- Yes, but this is not my main training

- No

7. Have you ever participated in an economic laboratory survey?

- No answer

- Yes

- No it's the first time

8. You have tested two choice methods on multiple questions: one where you vote only once for each of the two questions, and one where you vote in several stages. Which do you think is the most effective?

- No answer

- The one-step method

- The multi-step method

- Both methods are equally effective

- I don’t know / I prefer not to answer

9. Does the one-step method seem easy to understand and use?

- No answer

- Easy

- Rather easy

- Rather difficult

- Difficult

- I don’t know / I prefer not to answer 
10. Does the multi-step method seem easy to understand and use?

- No answer

- Easy

- Rather easy

- Rather difficult

- Difficult

- I don't know / I prefer not to answer 


\section{Screenshots}

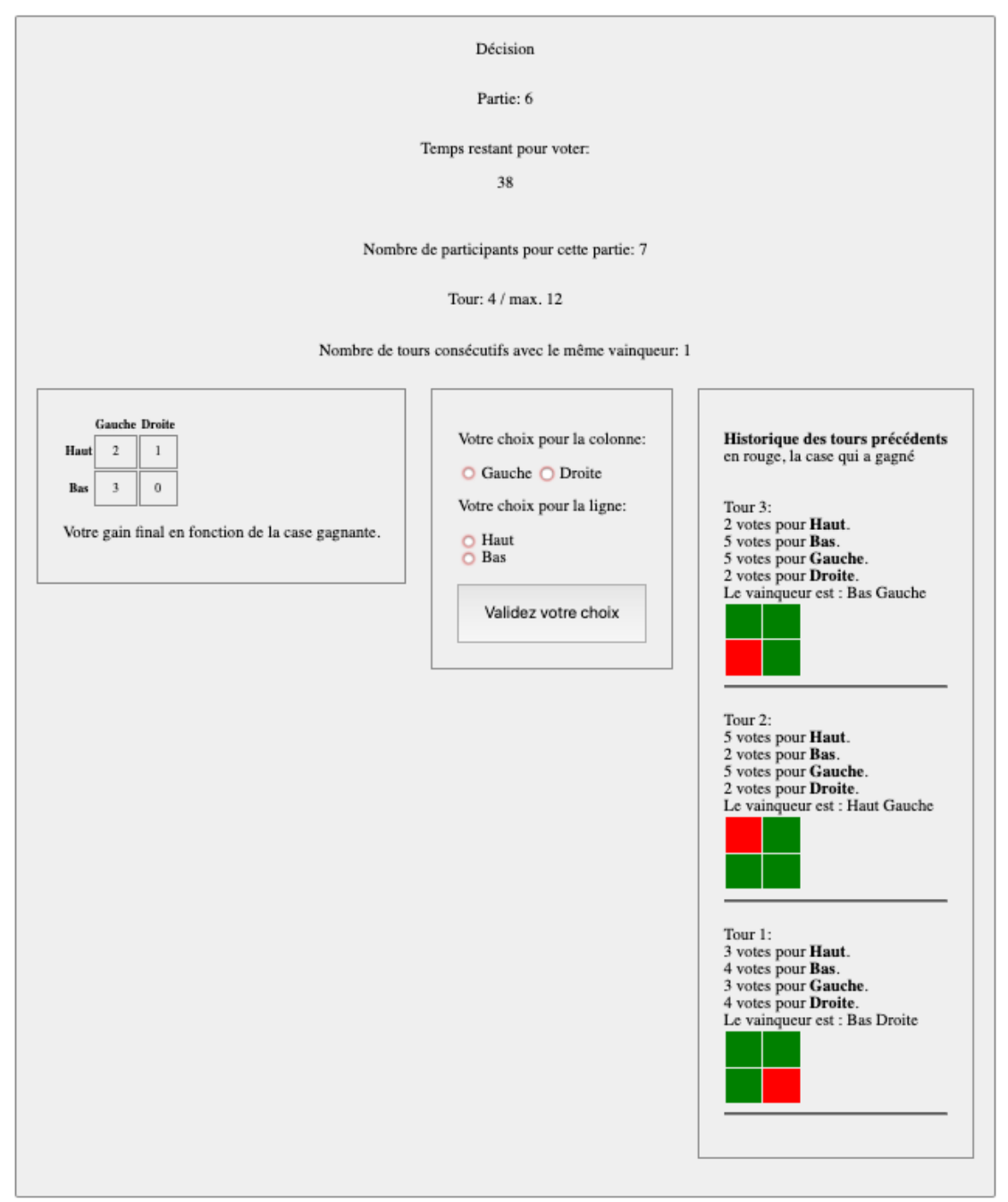

Figure 3: Fourth round in the sixth election. Note that the outcomes from past rounds appear on the right in reverse order. The subjects make their decisions in the middle area by choosing a row and a column and validating. 


\begin{tabular}{|c|c|c|c|}
\hline \multicolumn{4}{|c|}{$\begin{array}{l}\text { Résultat du tour } \\
\text { Partie: } 1\end{array}$} \\
\hline \multicolumn{4}{|c|}{$\begin{array}{l}\text { Votre gain final en fonction de la case gagnante: } \\
\text { Gauche Droite }\end{array}$} \\
\hline Haut & 1 & 0 & \\
\hline Bas & 3 & 2 & \\
\hline \multicolumn{4}{|c|}{ Les resultats du tour: } \\
\hline \multicolumn{4}{|c|}{$\begin{array}{l}0 \text { votes pour Haut. } \\
3 \text { votes pour Bas. } \\
\text { Résultat pour la ligne: Bas }\end{array}$} \\
\hline \multicolumn{4}{|c|}{$\begin{array}{l}1 \text { votes pour Gauche. } \\
2 \text { votes pour Droite. } \\
\text { Résultat pour la colonne: Droite }\end{array}$} \\
\hline \multicolumn{4}{|c|}{$\begin{array}{l}\text { Cette partie est terminée. } \\
\text { Votre gain pour cette partie est donc de } 2 € \text {. }\end{array}$} \\
\hline \multicolumn{4}{|c|}{ La prochaine partie débutera lorsque tous les autres participants seront prêts } \\
\hline OK & & & \\
\hline
\end{tabular}

Figure 4: Result screen after the first election. 